\title{
Age-dependent difference in impact of fertility preserving surgery on disease- specific survival in women with stage I borderline ovarian tumors
}

\author{
Haiyan Sun ${ }^{1,2}, X_{i}$ Chen ${ }^{1}$, Tao Zhu' ${ }^{1}$ Nanfang Liu', Aijun Yu ${ }^{1 *}$ and Shihua Wang ${ }^{3 *}$
}

\begin{abstract}
Background: This study was to determine age-specific impact of fertility preserving surgery on disease-specific survival in women with stage I borderline ovarian tumors (BOTs). Patients diagnosed during 1988-2000 were selected from The Surveillance, Epidemiology, and End Results (SEER) database. The age-specific impact of fertility preserving surgery and other risk factors were analyzed in patients with stage I BOTs using Cox proportion hazard regression models. Data from our hospital were collected during 1996-2017 to determine the prevalence of patients who had undergone fertility preserving surgery.

Results: Of a total 6295 patients in the SEER database, this study selected 2946 patients with stage T1 BOTs who underwent fertility preserving or radical surgery. Their median age at diagnosis was 45.0 years and the median follow-up time was 200 months. Fertility preserving surgery was performed in 1000/1751 (57.1\%) patients < 50 years and in 1,81/1195 (15.1\%) patients $\geq 50$ years. Fertility preserving surgery was significantly associated with worse disease-specific survival only in patients $\geq 50$ years. Increased age, stage T1c and mucinous histology were risk factors for overall patients or patients $\geq 50$ years, but not for $<50$ years. Data from our hospital showed that fertility preserving surgery was performed in 53.9 and $12.3 \%$ patients $<50$ and $\geq 50$ years with stage I disease, respectively.

Conclusion: Fertility preserving surgery is safe for women $<50$ years with early staged BOTs, but it may decrease diseasespecific survival in patients $\geq 50$ years. Conservative surgery is performed at a relatively high rate in patients $\geq 50$ years.
\end{abstract}

Keywords: Ovarian cancer, Borderline ovarian tumor, Fertility preserving surgery, Survival, Age, Histology, Stage

\section{Background}

Borderline ovarian tumors (BOTs) are histologically characterized as atypical epithelial proliferation without the presence of stromal invasion [1]. Serous and mucinous BOTs are the two major histological types [2]. These tumors have a low malignant potential to spread beyond the ovary with peritoneal involvement [1] and have an excellent prognosis [3-5]. This disease accounts for $10-15 \%$ of all epithelial ovarian cancers [6]. Compared to invasive epithelial ovarian

\footnotetext{
* Correspondence: yuaj@zjcc.org.cn; shwang@wakehealth.edu

'Department of Gynecologic Oncology, Zhejiang Cancer Hospital, 1 Banshan East Road, Zhejiang 310022, Hangzhou, China

${ }^{3}$ Department of Cancer Biology, Wake Forest School of Medicine, Winston Salem, NC 27157, USA

Full list of author information is available at the end of the article
}

cancers, BOTs occur more commonly, at an early stage, in women of childbearing ages [7].

The majority of BOTs are managed with surgery alone. Fertility preserving surgery is widely adopted for patients who have early-stage tumor development and a desire for fertility. Current consensus states that fertility preserving surgery is associated with an increased risk of recurrence [8-12]. Data from ours and other groups showed that certain styles of fertility preserving surgery may have a higher risk of recurrence than the others [13-15]. However, fertility preserving surgery was not shown to compromise overall survival in these patients [16-19].

Due to excellent prognosis, many patients with BOTs die due to other diseases. Overall survival is the 
end-point commonly used in previous studies to determine the impact of fertility preserving surgery; however, this may not accurately reflect the outcome of the surgery. Very few studies have investigated the impact of fertility preserving surgery on disease-specific survival [9]. Fertility preserving surgery has been shown age-dependent differences in its impact on recurrence free survival and other clinical outcomes in patients with BOTs [20]. Using a large population from a publicly available database, the objective of this study was to examine the age-specific impact of fertility preserving surgery on disease-specific survival in women with stage I BOTs.

\section{Methods}

The data for this study was obtained from the Surveillance, Epidemiology, and End Results (SEER) database maintained by the National Cancer Institute. This database collects information of cancer patients, which covers approximately $28 \%$ of the total US population. The SEER program statistical analysis software package (SEER*Stat version 8.3.4) was used to extract data from SEER18 Regs Research Data + Hurricane Katrina Impacted Louisiana Cases, Nov 2016 Sub (1973-2014 varying) [21]. BOTs in the SEER database between 1988 and 2000 were identified based upon the following histopathology codes: serous $8442-1,8451-1$ and 8462-1; and mucinous 8472-1, 8473-1 [22, 23].

Only patients with stage I BOTs with a record of survival times were included in this study. The status of oophorectomies and hysterectomies were quarried from codes in the site-specific surgery (1983-1997) and RX Summ-Surg Prim Site (1998+) (Additional file 1: Table S1). Fertility preserving surgery refers to preservation of the uterus and at least one side of a functional ovarium. This study thus defined the surgery as removal of the tumor or a unilateral oophorectomy without a hysterectomy. Radical surgery was defined as bilateral salpingo-oophorectomy with or without hysterectomy. Women were excluded if they did not receive surgery, their surgical status or survival time was unknown, or other surgical approaches were performed (Additional file 1: Table S1). The flow chart shows the detailed procedure for selecting patients (Additional file 1: Figure S1).

Variables extracted from the database were patients' demographics (age at diagnosis, ethnicities, marital status), surgery information (oophorectomy, hysterectomy, lymphadenectomy), tumor information (size, histology, stage), follow-up time and disease-specific death. Tumor stages were evaluated based on the American Joint Committee on Cancer (AJCC) 3rd staging classification [24].

To understand age-specific prevalence of fertility preserving surgery, women diagnosed with BOTs in Zhejiang Cancer Hospital during the year 1996-2017 were also included in this study. Tumor stages were evaluated based upon of the International Federation of Gynecology and Obstetrics (FIGO) 2014 classification system [25]. Stage T1 defined in AJCC 3rd is the same as stage I in FIGO 2014, except that stage Ic in FIGO 2014 is further divided into Ic1, Ic2 and Ic3 stages. The inclusion and exclusion criteria for these patients has been described previously [15].

Data were analyzed using SAS software V9.3 (SAS Institute, Inc., Cary, NC.). The ordinal/categorical data were examined using the $\chi^{2}$ test. Univariate or multivariate Cox proportional hazards models were used to determine the impacts of fertility preserving surgery and other risk factors on disease-specific survival. The Kaplan-Meier survival curves were generated and their significant differences were analyzed by log-rank tests. Two-sided $P$ values less than 0.05 were considered statistically significant.

\section{Results}

A total of 6295 women with BOTs were initially identified from the SEER database. Based on our inclusion and exclusion criteria, a total of 2946 cases with stage I BOTs were included in this study. The detailed demographic information and pathoclinical features are listed in Table 1. The mean age of these patients was $47.1 \pm 17.0$ years with a median age of 45.0 years (range 10-96 years). The median follow-up time was 200 months (range 1-323 months). Within this population, $59.4 \%(n=1751)$ were $<50$ years old and $40.6 \%(n=1195)$ were $\geq 50$ years. Most patients $(85.0 \%)$ studied were Caucasian. The majority of BOTs were diagnosed at stage $\mathrm{T} 1 \mathrm{a}(79.3 \%)$. Fertility preserving surgery was performed in 1181 (40.1\%) patients. Hysterectomy and recorded lymphadenectomy were performed in 1374 (47.6\%) and 341 (11.4\%) patients, respectively. At the end of the follow-up year, 70 (2.4\%) patients died from this disease.

The characteristics of patients in two age groups $(<50$ and $\geq 50$ years) are presented in Table 1 . Compared to patients $<50$ years, patients $\geq 50$ years underwent fertility preserving surgery less frequently $(15.1 \%$ vs $57.1 \%, P<0.0001)$. A higher proportion of them were Caucasian $(87.2 \%$ vs $83.6 \%, P=0.0049)$, underwent hysterectomy $(63.7 \%$ vs $35.0 \%, P<0.0001)$ and lymphadenectomy $(14.0 \%$ vs $9.6 \%, P=0.0050)$. They had a higher rate of disease-specific death $(4.5 \%$ vs $0.9 \%, P<0.0001)$, but a shorter mean follow-up time $(163.4 \pm 77.8$ vs $215.0 \pm$ 59.2 months, $P<0.0001$ ).

The features of patients were compared between those who underwent fertility preserving surgery vs. radical surgery. Of the entire population studied, including both age groups, married patients and patients with serous tumors at stage $\mathrm{T} 1 \mathrm{~b}$ or $\mathrm{T} 1 \mathrm{c}$ were less likely to undergo fertility preserving surgery. Patients receiving fertility 
Table 1 Demographic and pathoclinical features of BOT patients

\begin{tabular}{|c|c|c|c|c|c|}
\hline Variables & & Overall $(n=2946)$ & $<50(n=1751)$ & $\geq 50(n=1195)$ & $P$ value \\
\hline \multicolumn{6}{|l|}{ Age (years) } \\
\hline & Median (range) & $45.0(10-96)$ & $36.0(10-49)$ & $64.0(50-96)$ & \\
\hline & Mean \pm SD & $47.1 \pm 17.0$ & $35.3 \pm 8.6$ & $64.3 \pm 10.2$ & \\
\hline \multicolumn{6}{|l|}{ Race } \\
\hline & White & $2505(85.0)$ & $1463(83.6)$ & $1042(87.2)$ & 0.0049 \\
\hline & Black & $170(5.8)$ & $102(5.8)$ & $68(5.7)$ & \\
\hline & Others & $271(9.2)$ & $186(10.6)$ & $85(7.1)$ & \\
\hline \multicolumn{6}{|l|}{ Histology } \\
\hline & Serous & $1646(55.9)$ & $961(54.9)$ & $685(57.3)$ & 0.1905 \\
\hline & Mucinous & $1300(44.1)$ & $790(45.1)$ & $510(42.7)$ & \\
\hline \multicolumn{6}{|l|}{ Marital status } \\
\hline & Single* & $1268(43.0)$ & $736(42.0)$ & $532(44.5)$ & 0.3188 \\
\hline & Married & $1560(53.0)$ & $940(53.7)$ & $620(51.9)$ & \\
\hline & Unknown & $118(4.0)$ & $75(4.3)$ & $43(3.6)$ & \\
\hline \multicolumn{6}{|c|}{ Lymphadenectomy } \\
\hline & No & $2602(88.3)$ & $1575(90.0)$ & $1027(85.9)$ & 0.0004 \\
\hline & Yes & $336(11.4)$ & 169 (9.6) & $167(14.0)$ & \\
\hline & Unknown & $8(0.3)$ & $7(0.4)$ & $1(0.1)$ & \\
\hline \multicolumn{6}{|l|}{ AJCC stage } \\
\hline & T1a & $2337(79.3)$ & $1407(80.4)$ & $930(77.8)$ & 0.0582 \\
\hline & $\mathrm{T} 1 \mathrm{~b}$ & $177(6.0)$ & $90(5.1)$ & $87(7.3)$ & \\
\hline & T1c & 281 (9.6) & $171(9.8)$ & $110(9.2)$ & \\
\hline & $\mathrm{T} 1 \mathrm{x}$ & $151(5.1)$ & $83(4.7)$ & $68(5.7)$ & \\
\hline \multicolumn{6}{|l|}{ Tumor size } \\
\hline & $\leq 5 \mathrm{~cm}$ & $425(40.6)$ & $244(40.3)$ & $181(40.9)$ & 0.8402 \\
\hline & $>5 \mathrm{~cm}$ & $622(59.4)$ & $361(59.7)$ & $261(59.1)$ & \\
\hline \multirow[t]{2}{*}{ Hysterectomy } & No & $1572(53.4)$ & $1138(65.0)$ & $434(36.3)$ & $<0.0001$ \\
\hline & Yes & $1374(47.6)$ & $613(35.0)$ & $761(63.7)$ & \\
\hline \multicolumn{6}{|c|}{ Fertility preserving surgery } \\
\hline & No & 1765 (59.9) & $751(42.9)$ & $1014(84.9)$ & $<0.0001$ \\
\hline & Yes & $1181(40.1)$ & $1000(57.1)$ & $181(15.1)$ & \\
\hline \multicolumn{6}{|l|}{ Laterality } \\
\hline & Unilateral & $1092(37.1)$ & $646(36.9)$ & $446(37.3)$ & 0.9652 \\
\hline & Bilateral & $1253(42.5)$ & $748(42.7)$ & $505(42.3)$ & \\
\hline & Unknown & $601(20.4)$ & $357(20.4)$ & $244(20.4)$ & \\
\hline \multirow[t]{2}{*}{ Death } & No & $2876(97.6)$ & $1735(99.1)$ & $1141(95.5)$ & $<0.0001$ \\
\hline & Yes & $70(2.4)$ & $16(0.9)$ & $54(4.5)$ & \\
\hline \multicolumn{6}{|c|}{ Follow-up time (months) } \\
\hline & Median (range) & $200(1-323)$ & $217(1-323)$ & $176(1-323)$ & \\
\hline & Mean \pm SD & $194.0 \pm 72.0$ & $215.0 \pm 59.2$ & $163.4 \pm 77.8$ & $<0.0001$ \\
\hline
\end{tabular}

*including never married, divorced, widowed. Abbreviations: AJCC, American Joint Commission on Cancer; T1x, T1 undefined 
preserving surgery were less likely to undergo lymphadenectomy. Caucasian patients, both in the entire population, as well as in the $<50$ age group were less likely to undergo fertility preserving surgery (Table 2 ).

Results of univariate and multivariate analysis of disease-specific survival in the whole population are presented in Table 3. Increased age (hazard ratio $(\mathrm{HR})=1.06$, 95\% confidence interval $(\mathrm{CI}): 1.04-1.08$, $P<0.0001$ ), stage T1c (vs T1a, HR $=2.42,95 \%$ CI: $1.30-4.48$, $P=0.0051$ ) were significantly associated with worse disease-specific survival. Without controlling of other confounding factors, fertility preserving surgery (vs radical surgery, $\mathrm{HR}=0.52,95 \% \mathrm{CI}$ : $0.30-0.88, P=0.0142$ ) was associated with improved disease-specific survival. The survival curves are presented at Additional file 1: Figure S2A and S2B. Multivariate analysis showed that increased age (HR $=1.06,95 \%$ CI: $1.05-1.08, P<0.0001)$, stage T1b (vs T1a, HR $=2.38,95 \% \mathrm{CI}: 1.05-5.39, P=0.0369$ ), stage T1c (vs T1a, HR $=3.00,95 \%$ CI: $1.60-5.65, P=0.0006$ ) and mucinous histology ( $\mathrm{HR}=1.73,95 \% \mathrm{CI}: 1.06-2.83$, $P=0.0285)$ were significantly associated with worse disease-specific survival, whereas fertility preserving

Table 2 Features of patients who underwent fertility preserving surgery (Yes) or radical surgery (No)

\begin{tabular}{|c|c|c|c|c|c|c|c|c|c|c|}
\hline \multirow{2}{*}{\multicolumn{2}{|c|}{$\begin{array}{l}\text { Variables } \\
\text { Fertility preserving surgery }\end{array}$}} & \multicolumn{3}{|c|}{ Total $(n=2946)$} & \multicolumn{3}{|c|}{$<50(n=1751)$} & \multicolumn{3}{|c|}{$\geq 50(n=1195)$} \\
\hline & & \multirow[t]{2}{*}{ Yes } & \multirow[t]{2}{*}{ No } & \multirow[t]{2}{*}{$P$ values } & \multirow[t]{2}{*}{ Yes } & \multirow[t]{2}{*}{ No } & \multirow[t]{2}{*}{$P$ values } & \multirow[t]{2}{*}{ Yes } & \multirow[t]{2}{*}{ No } & \multirow[t]{2}{*}{$P$ values } \\
\hline Race & & & & & & & & & & \\
\hline & White & 964 (81.6) & 1541 (87.3) & $<0.0001$ & 806 (80.6) & $657(87.5)$ & $<0.0001$ & $158(87.3)$ & $884(87.2)$ & 0.0952 \\
\hline & Black & $75(6.4)$ & $95(5.4)$ & & $60(6.0)$ & $42(5.6)$ & & $15(8.3)$ & $53(5.2)$ & \\
\hline & Other & $142(12.0)$ & $129(7.3)$ & & $134(72.0)$ & $52(6.9)$ & & $8(4.4)$ & $77(7.6)$ & \\
\hline \multicolumn{11}{|c|}{ Marital status } \\
\hline & Single* & $580(49.1)$ & $688(39.0)$ & $<0.0001$ & $487(48.7)$ & $249(33.2)$ & $<0.0001$ & $93(51.4)$ & 439 (43.3) & 0.0220 \\
\hline & Married & 555 (35.6) & 1005 (56.9) & & $477(47.7)$ & $463(61.6)$ & & $78(43.1)$ & $542(53.4)$ & \\
\hline & Unknown & $46(3.9)$ & $72(4.1)$ & & $36(3.6)$ & $39(5.2)$ & & $10(5.5)$ & $33(3.3)$ & \\
\hline \multicolumn{11}{|c|}{ Histology } \\
\hline & Serous & 615 (52.1) & $1031(58.4)$ & 0.0007 & $514(51.4)$ & $447(59.5)$ & 0.0007 & 101 (55.8) & $584(57.6)$ & 0.6533 \\
\hline & Mucinous & $566(47.9)$ & 734 (41.6) & & $486(48.6)$ & $304(40.5)$ & & $80(44.2)$ & $430(42.4)$ & \\
\hline \multicolumn{11}{|c|}{ AJCC stage } \\
\hline & T1a & $1000(84.7)$ & 1337 (75.6) & $<0.0001$ & $850(85.0)$ & $557(74.2)$ & $<0.0001$ & $150(82.9)$ & $780(76.9)$ & 0.0008 \\
\hline & $\mathrm{T} 1 \mathrm{~b}$ & $22(1.9)$ & 155 (8.8) & & $21(2.1)$ & $69(9.2)$ & & $1(0.6)$ & $86(8.5)$ & \\
\hline & T1c & 101 (8.6) & $180(10.2)$ & & $86(8.6)$ & $85(11.3)$ & & $15(8.3)$ & $95(9.4)$ & \\
\hline & $\mathrm{T} 1 \mathrm{x}$ & $58(4.9)$ & $93(5.3)$ & & $43(4.3)$ & $40(5.3)$ & & $15(8.3)$ & $53(5.2)$ & \\
\hline \multicolumn{11}{|c|}{ Hysterectomy } \\
\hline & No & $1181(100)$ & 391 (22.2) & $<0.0001$ & $100(100)$ & $138(18.4)$ & $<0.0001$ & $181(100)$ & $253(25.0)$ & $<0.0001$ \\
\hline & Yes & $0(0)$ & $1374(77.8)$ & & $0(0)$ & $631(81.6)$ & & $0(0)$ & $761(75.0)$ & \\
\hline \multicolumn{11}{|c|}{ Tumor size } \\
\hline & $<=5$ & $166(40.3)$ & $259(40.8)$ & 0.8731 & $139(39.8)$ & $105(41.0)$ & 0.7686 & $27(42.9)$ & $154(40.6)$ & 0.7396 \\
\hline & $>5$ & $246(59.7)$ & $376(59.2)$ & & $210(60.2)$ & $151(59.0)$ & & $36(57.1)$ & $225(59.4)$ & \\
\hline \multicolumn{11}{|c|}{ Lymphadenectomy } \\
\hline & No & 1085 (91.9) & $1517(86.0)$ & $<0.0001$ & $1032(61.3)$ & $652(38.7)$ & 0.0001 & $161(89.0)$ & $866(85.4)$ & 0.4254 \\
\hline & Yes & $91(7.8)$ & $245(13.9)$ & & $80(44.7)$ & 99 (55.3) & & $20(11.0)$ & $147(14.5)$ & \\
\hline & Unknown & $5(0.4)$ & $3(0.2)$ & & $5(71.4)$ & $2(28.6)$ & & $0(0)$ & $1(0.1)$ & \\
\hline \multicolumn{11}{|c|}{ Laterality } \\
\hline & Unilateral & $426(36.1)$ & $666(37.7)$ & 0.6573 & $372(37.2)$ & $274(36.5)$ & 0.8792 & $54(29.8)$ & $392(38.7)$ & 0.0679 \\
\hline & Bilateral & $510(43.2)$ & $743(42.1)$ & & $422(42.2)$ & $326(43.4)$ & & $88(48.6)$ & $417(41.1)$ & \\
\hline & Unknown & $245(20.7)$ & $356(20.2)$ & & $206(20.6)$ & $151(20.1)$ & & 39 (21.6) & $205(20.2)$ & \\
\hline \multirow[t]{2}{*}{ Death } & No & $1162(98.4)$ & $1718(97.1)$ & 0.0253 & $994(99.4)$ & $741(98.7)$ & 0.1113 & $168(92.8)$ & $973(96.0)$ & 0.0611 \\
\hline & Yes & $19(1.6)$ & $51(2.9)$ & & $6(0.6)$ & $10(1.3)$ & & $13(7.2)$ & $41(4.0)$ & \\
\hline
\end{tabular}


Table 3 Survival analysis of cancer specific survival in the whole population

\begin{tabular}{|c|c|c|c|c|c|}
\hline \multirow[t]{2}{*}{ Variables } & & \multicolumn{2}{|l|}{ Univariate } & \multicolumn{2}{|l|}{ Multivariate } \\
\hline & & $\mathrm{HR}(95 \% \mathrm{Cl})$ & $P$ values & $\mathrm{HR}(95 \% \mathrm{Cl})$ & $P$ values \\
\hline Age & & $1.06(1.04-1.08)$ & $<0.0001$ & $1.06(1.05-1.08)$ & $<0.0001$ \\
\hline \multirow[t]{5}{*}{ AJCC stage } & & & & 1 & \\
\hline & T1a & 1 & & & \\
\hline & $\mathrm{T} 1 \mathrm{~b}$ & $2.22(1.00-4.92)$ & 0.0503 & $2.38(1.05-5.39)$ & 0.0369 \\
\hline & T1c & $2.42(1.30-4.48)$ & 0.0051 & $3.00(1.60-5.65)$ & 0.0006 \\
\hline & $\mathrm{T} 1 \mathrm{x}$ & $1.58(0.63-3.98)$ & 0.3318 & $1.45(0.57-3.67)$ & 0.4349 \\
\hline \multicolumn{6}{|l|}{ Histology } \\
\hline & Serous & 1 & & 1 & \\
\hline & Mucinous & $1.40(0.88-2.24)$ & 0.1600 & $1.73(1.06-2.83)$ & 0.0285 \\
\hline \multicolumn{6}{|l|}{ Race } \\
\hline & White & 1 & & & \\
\hline & Black & $0.75(0.24-2.40)$ & 0.6309 & & \\
\hline & Other & $0.57(0.13-1.35)$ & 0.1479 & & \\
\hline \multicolumn{6}{|c|}{ Marital status } \\
\hline & Single & 1 & & & \\
\hline & Married & $0.82(0.52-1.32)$ & 0.4191 & & \\
\hline Unknown & & 0 & 0.9831 & & \\
\hline \multicolumn{6}{|c|}{ Fertility preserving Surgery } \\
\hline & No & 1 & & & \\
\hline & Yes & $0.52(0.31-0.88)$ & 0.0142 & & \\
\hline \multicolumn{6}{|c|}{ Hysterectomy } \\
\hline & No & 1 & & & \\
\hline & Yes & $1.04(0.65-1.66)$ & 0.8755 & & \\
\hline \multicolumn{6}{|l|}{ Tumor size } \\
\hline & $<=5$ & 1 & & & \\
\hline & $>5$ & $1.47(0.55-3.92)$ & 0.4438 & & \\
\hline \multicolumn{6}{|c|}{ Lymphadenectomy } \\
\hline & No & 1 & & & \\
\hline & Yes & $0.77(0.33-1.77)$ & 0.5330 & & \\
\hline & Unknown & 0 & 0.9854 & & \\
\hline \multicolumn{6}{|l|}{ Laterality } \\
\hline & Unilateral & 1 & & & \\
\hline & Bilateral & $1.13(0.767-1.93)$ & 0.6432 & & \\
\hline & Unknown & $1.13(0.59-2.14)$ & 0.7208 & & \\
\hline
\end{tabular}

Abbreviations: AJCC, American joint commission on Cancer; T1x, T1 undefined

surgery is not a factor significantly related to disease-specific death.

We further preformed survival analysis for patients in $<50$ and $\geq 50$ age groups. In patients $<50$ years old, only the undefined $\mathrm{T} 1$ stage (vs $\mathrm{T} 1 \mathrm{a}, \mathrm{HR}=5.99$, 95\% CI: $1.59-22.60, P=0.0082$ ) was significantly associated with poorer disease-specific survival. No other significant risk factors were observed in these patients using univariate analysis. No risk factors were correlated with disease-specific survival using multivariate analysis (Table 4 ). In patients $\geq 50$ years, univariate analysis showed that increased age $(\mathrm{HR}=1.04,95 \% \mathrm{CI}: 1.01-1.07$, $P=0.0063)$, fertility preserving surgery $(\mathrm{HR}=2.04,95 \%$ CI: $1.09-3.81, P=0.0251$ ), stage T1c (vs T1a, $\mathrm{HR}=2.38$, 95\% CI: $1.18-4.78, P=0.0151)$ and hysterectomy $(\mathrm{HR}=0.41$, 95\% CI: $0.24-0.70, P=0.0012$ ) were risk factors significantly associated with disease-specific survival (Table 5). Disease-specific survival curves of the above risk factors are 
Table 4 Univariate survival analysis in patients of age $<50$ years

\begin{tabular}{|c|c|c|c|}
\hline Variables & & $\mathrm{HR}(95 \% \mathrm{Cl})$ & $P$ values \\
\hline Age & & $1.04(0.98-1.11)$ & 0.2070 \\
\hline Race & & & \\
\hline & White & 1 & \\
\hline & Black & 0 & 0.9908 \\
\hline & Other & $1.19(0.27-5.25)$ & 0.8173 \\
\hline Marital status & & & \\
\hline & Single* & 1 & \\
\hline & Married & $1.66(0.58-4.79)$ & 0.3467 \\
\hline & unknown & 0 & 0.9920 \\
\hline Histology & Serous & & \\
\hline & Mucinous & $1.20(0.45-3.19)$ & 0.7200 \\
\hline AJCC stage & & & \\
\hline & T1a & 1 & \\
\hline & $\mathrm{T} 1 \mathrm{~b}$ & $4.12(0.87-19.41)$ & 0.0734 \\
\hline & T1c & $3.07(0.81-11.57)$ & 0.0979 \\
\hline & $\mathrm{T} 1 \mathrm{x}$ & $5.99(1.59-22.60)$ & 0.0082 \\
\hline Fertility preserving s & & & \\
\hline & No & 1 & \\
\hline & Yes & $0.46(0.17-1.28)$ & 0.1374 \\
\hline Hysterectomy & & & \\
\hline & No & 1 & \\
\hline & Yes & $2.26(0.84-6.07)$ & 0.1061 \\
\hline Tumor size & $\leq 5 \mathrm{~cm}$ & 1 & \\
\hline & $>5 \mathrm{~cm}$ & $0.75(0.110-5.40)$ & 0.7771 \\
\hline Lymphadenectomy & No & 1 & \\
\hline & Yes & 0 & 0.9922 \\
\hline & Unknown & 0 & 0.9986 \\
\hline Lymph node numbe & & & \\
\hline & $1-10$ & 1 & \\
\hline & $>10$ & 0 & 0.9911 \\
\hline & Unknown & 0 & 0.9906 \\
\hline Laterality & Unilateral & 1 & \\
\hline & Bilateral & $150(0.44-5.12)$ & 0.5183 \\
\hline & Unknown & $2.23(0.60-8.31)$ & 0.2314 \\
\hline
\end{tabular}

${ }^{*}$ including never married, divorced, widowed. Abbreviations: AJCC, American Joint Commission on Cancer; T1x, T1 undefined

presented at Fig. 1a, b and c. Multivariate analysis showed that the increased age $(\mathrm{HR}=1.04,95 \% \mathrm{CI}: 1.01-1.07$, $P=0.0108)$, fertility preserving surgery $(\mathrm{HR}=1.99$, 95\% CI: $1.059-3.77, P=0.0253)$, stage T1c $(\mathrm{HR}=2.87$, 95\% CI: $1.41-5.86, P=0.0037)$ and mucinous histology $(\mathrm{HR}=1.87, \quad 95 \%$ CI: $1.07-3.27, \quad P=0.0278)$ were risk factors significantly associated with worse disease-specific survival (Table 5).
Data from our hospital showed that 255 women with BOTs underwent surgery from 1996 to 2017. The median age was 42 years (range 15-87). Among these patients, 108 (42.4\%) had serous tumors and 118 (46.3\%) had mucinous tumors. A total of 170 (66.7\%) cases were stage I, with one case having an unknown age. Fertility preserving surgery was performed in 113 overall (44.3\%) patients (Additional file 1: Table S2). The rate of fertility preserving surgery performed in these patients at stage I was further analyzed after dividing them into two age groups ( $<50$ and $\geq 50$ years). Our result showed that $56 /$ $104(53.9 \%)$ patients $<50$ and $8 / 65(12.3 \%)$ patients $\geq 50$ underwent fertility preserving surgery. These two groups had other similar pathoclinical features (Table 6).

\section{Discussion}

With a sample size of 2946 patients and a median follow-up time of 200 months, this study examined age-specific impact of fertility preserving surgery on disease-specific survival in women with T1 BOTs. The main finding of this study was that fertility preserving surgery was significantly associated with worse disease-specific survival only in patients $\geq 50$ years, but not in overall patients or patients $<50$ years. Our results revealed an age-dependent difference in impact of fertility preserving surgery on disease-specific survival in these patients. This finding suggests that while conservative surgery may comprise survival in women $\geq 50$ years, it is safe for patients $<50$ years. Future studies with randomized clinical trials are warranted to verify this finding.

Previous studies have consistently shown that fertility preserving surgery may increase the risk of recurrence $[11,14,15,26]$. Interestingly, the risk of recurrence was higher in younger patients with BOTs $[9,11,18,20]$. Most of the recurrences showed no malignant transformation and were curable by a single surgery without compromising overall survival $[9,16-18]$. Invasive carcinoma diagnosed in recurrences $[9,11,18,27-29]$ is the cause of cancer deaths [30]. A sub-analysis of the Arbeitsgemeinschaft Gynaekologische Onkologie (AGO) ROBOT study evaluated data from a total of 950 patients with BOTs. Their results showed that $66.7 \%$ of recurrent diseases were invasive carcinoma in patients $\geq 40$ years, which dramatically contrasted with a recurrence of $12 \%$ of invasive carcinomas in patients $<40$ years [20]. The increased incidence of invasive recurrent ovarian cancer in older patients may account for the reduced disease-specific survival after fertility preserving surgery.

This study is unable to address the molecular mechanism whereby fertility preserving surgery is associated with reduced disease-specific survival in patients $\geq 50$ years. Akeson et al. [7] reported patients $>60$ had significantly more aneuploid tumors. Aneuploidy was 
Table 5 Survival analysis in patients $\geq 50$ years

\begin{tabular}{|c|c|c|c|c|c|}
\hline \multirow[t]{2}{*}{ Variables } & & \multicolumn{2}{|l|}{ Univariate } & \multicolumn{2}{|l|}{ Multivariate* } \\
\hline & & $\mathrm{HR}(95 \% \mathrm{Cl})$ & $P$ values & $\mathrm{HR}(95 \% \mathrm{Cl})$ & $P$ values \\
\hline Age & & $1.04(1.01-1.07)$ & 0.0063 & $1.04(1.01-1.07)$ & 0.0108 \\
\hline \multicolumn{6}{|c|}{ Fertility preserving surgery } \\
\hline & No & 1 & & 1 & \\
\hline & Yes & $2.04(1.09-3.81)$ & 0.0251 & $1.99(1.05-3.77)$ & 0.0347 \\
\hline \multicolumn{6}{|c|}{ AJCC stage } \\
\hline & T1a & 1 & & 1 & \\
\hline & $\mathrm{T} 1 \mathrm{~b}$ & $1.56(0.61-3.96)$ & 0.3531 & $2.30(0.87-6.09)$ & 0.0931 \\
\hline & T1c & $2.38(1.18-4.78)$ & 0.0151 & $2.87(1.41-5.86)$ & 0.0037 \\
\hline & $\mathrm{T} 1 \mathrm{x}$ & $0.60(0.14-2.49)$ & 0.4793 & $0.58(0.14-2.42)$ & 0.4527 \\
\hline \multicolumn{6}{|l|}{ Histology } \\
\hline & Serous & 1 & & 1 & \\
\hline & Mucinous & $1.53(0.90-2.62)$ & 0.1175 & $1.87(1.07-3.27)$ & 0.0278 \\
\hline \multicolumn{6}{|l|}{ Race } \\
\hline & White & 1 & & & \\
\hline & Black & $1.01(0.32-3.24)$ & 0.9879 & & \\
\hline & Other & $0.23(0.03-1.68)$ & 0.1476 & & \\
\hline \multicolumn{6}{|c|}{ Marital status } \\
\hline & Single ${ }^{*}$ & 1 & & & \\
\hline & Married & $0.63(0.37-1.08)$ & 0.0915 & & \\
\hline & unknown & $\mathrm{N} / \mathrm{A}$ & 0.9860 & & \\
\hline \multicolumn{6}{|c|}{ Hysterectomy } \\
\hline & No & 1 & & & \\
\hline & Yes & $0.41(0.24-0.70)$ & 0.0012 & & \\
\hline \multicolumn{6}{|l|}{ Size $(\mathrm{cm})$} \\
\hline & $<=5$ & 1 & & & \\
\hline & $>5$ & $1.00(1.00-1.01)$ & 0.3384 & & \\
\hline \multicolumn{6}{|c|}{ Lymphadenectomy } \\
\hline & No & 1 & & & \\
\hline & Yes & $0.78(0.33-1.82)$ & 0.5639 & & \\
\hline & Unknown & 0 & 0.9888 & & \\
\hline \multicolumn{6}{|l|}{ Laterality } \\
\hline & Unilateral & & & & \\
\hline & Bilateral & $1.10(0.61-1.99)$ & 0.7511 & & \\
\hline & Unknown & $0.91(0.42-1.94)$ & 0.7986 & & \\
\hline
\end{tabular}

*including never married, divorced, widowed. Abbreviations: AJCC, American Joint Commission on Cancer; T1x, T1 undefined

associated with an increased mortality of patients with BOTs [31]. Furthermore, BRAF, KRAS and other mutations, and ERBB2 overexpression/amplification were frequently observed BOTs [32-34]. It is unknown whether age-related changes in DNA ploidy and gene mutations play a role in increased invasive recurrence in older patients.

It is noted that as high as $15.1 \%$ patients $\geq 50$ years with stage I BOTs underwent fertility preserving surgery in this selected population. Reports are still sparse regarding the prevalence of patients undergoing fertility preserving surgery within different age groups. Trillsch et al. reported that fertility preserving surgery was carried out in $53.2 \%(149 / 280)$ of patients < 40 years, $2.8 \%(19 / 670)$ of overall patients $\geq 40$ years with BOTs [20]. It is speculated that a higher rate of conservative surgery was performed in their patients with stage I BOTs. Comparable to the result from the SEER database, 

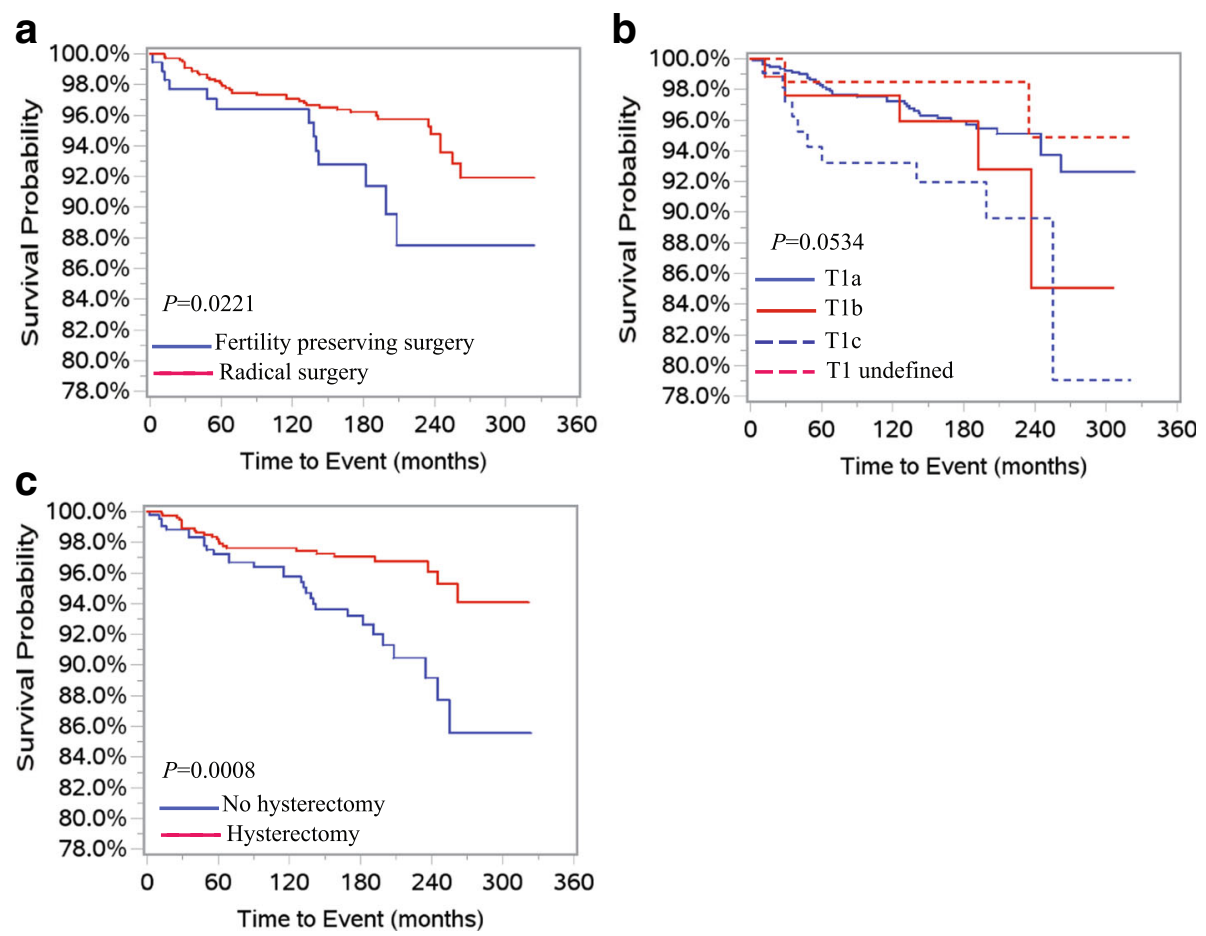

Fig. 1 Kaplan-Meier survival curves for patients $\geq 50$ with stage I borderline ovarian tumors. a Fertility preserving surgery vs radical surgery. b Sub-stages. c Hysterectomy status

data from our hospital showed $12.3 \%$ women $\geq 50$ years with stage I disease underwent fertility preserving surgery. Women $\geq 50$ years lose reproductive ability. Preservation of fertility is therefore not the primary objective when adopting conservative surgery in these patients. Conservative surgery brings less postoperative morbidities. Specific reasons older patients undergo conservative surgery remain unknown. Based upon the findings of this study, these patients may need extra attention after conservative surgery.

Our study also identified that increased age, a higher stage (T1c) and mucinous histology were significantly associated with decreased disease-specific survival in overall patients or patients $\geq 50$. Using the same database, a previous study revealed that older age $(\geq 50)$, higher stage and mucinous histology were associated with worse disease-specific survival in patients with stage I BOTs [23]. The tumor stage is a known prognostic factor for patients with BOTs [29]. Our results further revealed that higher stage (T1c) was significantly associated with poorer disease-specific survival in BOT patients at the early stage. Patients with mucinous BOTs were reported to have a worse prognosis compared with to patients with serous BOTs [31, 35]. The worse survival is partially explained by a higher incidence of invasive recurrent carcinoma in patients with mucinous
BOTs. Karlsen et al. [9] found that 6 out of 7 invasive recurrences were patients with mucinous BOTs at FIGO stage I.

An earlier study identified 6017 cases of BOTs from the SEER database. Their results revealed that the lymph node involvement was not significantly associated with disease-specific survival after adjusting with FIGO stages [36]. No impact of lymph node involvement on overall survival in patients with BOT were also observed in other studies [37, 38]. Data from our work and the previous study [23] showed that lymphadenectomy were not a risk factor associated with disease-specific survival.

The use of this database has numerous limitations. Patients were included retrospectively and were not randomly assigned to a treatment. Detailed information of fertility preserving surgery is unavailable. Among patients with stage I disease, $41.6 \%$ (2118/5094) were excluded from the study due to unclear surgical information. Many important pathological features of the tumors, such as invasive implants, and micropapillary patterns, are unavailable in these patients. Ovarian cancer related blood biomarkers were not recorded in the SEER database. It is unknown whether there have been recurrences and the types of relapses may have occurred in these patients. The location of harvested lymph nodes are not defined and their numbers are missing in some 
Table 6 Pathoclinical features of patients with stage I borderline ovarian tumor from Zhejiang Cancer Hospital

\begin{tabular}{|c|c|c|c|c|}
\hline \multirow[t]{2}{*}{ Variables } & & \multicolumn{2}{|l|}{ Age (yeas) } & \multirow[b]{2}{*}{$P$ values } \\
\hline & & $<50$ & $\geq 50$ & \\
\hline \multicolumn{5}{|c|}{ Fertility preservation surgery } \\
\hline & No & $48(46.1)$ & $57(87.7)$ & \multirow[t]{2}{*}{$<0.0001$} \\
\hline & Yes & $56(53.9)$ & $8(12.3)$ & \\
\hline \multicolumn{5}{|l|}{ Histology } \\
\hline & Serous & $43(41.3)$ & $27(32.3)$ & \multirow[t]{4}{*}{0.9774} \\
\hline & Mucinous & $52(50.0)$ & $39(60.0)$ & \\
\hline & Endometrioid & $7(7.7)$ & $4(6.1)$ & \\
\hline & Clear cells & $1(1.0)$ & $1(1.5)$ & \\
\hline \multicolumn{5}{|c|}{ FIGO stage } \\
\hline & IA & $70(67.3)$ & $42(64.6)$ & \multirow[t]{3}{*}{0.9263} \\
\hline & $\mathrm{IB}$ & $11(10.6)$ & $7(10.8)$ & \\
\hline & IC & $23(22.1)$ & $16(24.6)$ & \\
\hline \multicolumn{5}{|c|}{ Tumor size (cm) } \\
\hline & $\leq 5$ & $42(40.4)$ & $21(32.3)$ & \multirow[t]{2}{*}{0.2908} \\
\hline & $>5$ & $62(59.6)$ & $44(67.6)$ & \\
\hline \multicolumn{5}{|l|}{ Laterality } \\
\hline & Unilateral & $80(82.7)$ & $49(76.9)$ & \multirow[t]{2}{*}{0.3574} \\
\hline & Bilateral & $24(17.3)$ & $16(23.1)$ & \\
\hline \multicolumn{5}{|l|}{ Death } \\
\hline & No & $103(99.0)$ & $64(98.5)$ & \multirow[t]{2}{*}{0.6938} \\
\hline & Yes & $1(1.0)$ & $1(1.5)$ & \\
\hline
\end{tabular}

patients. Many other limitations using the SEER database have been addressed in a previous study [23].

Use of the SEER database in this study had its strength in its relatively large sample size, long follow-up time, and particularly, relatively large number of disease-specific deaths. Using the same database, the previous study identified 4943 cases with stage T1 BOTs from the same database, and reported a total of 159 (3.2\%) deaths in a median follow-up time of 187 months [23]. In contrast, the number of disease-specific deaths reported in previous studies was limited. A cohort included 1143 BOT patients with 1005 (87.9\%) patients at FIGO stage I. During a median follow-up time of 49.9 months (range 3.5-99 months), only $7(0.6 \%)$ patients I died of this disease [9]. In another study, a total of 151 patients were recruited. Among them, 87 (64.4\%) patients were at FIGO stage I, and 113 patients (74.8\%) had follow-up information. After a median follow-up time of 86 (range 0.1-432) months, 7 (6.2\%) patients died of this disease [39]. A multi-center study included 457 patients with $390(85.3 \%)$ at stage I. During a mean follow-up of 88.3 months, 9 (2\%) patients died of this disease [40]. Leake et al. reported 13 (6.5\%) disease-specific deaths in a cohort of 200 patients in a median follow-up time of 120 months [41].

\section{Conclusion}

It is safe to perform fertility preserving surgery for women of child-bearing age with stage I BOTs. This surgery may increase the risk of disease-specific death for women of older ages ( $\geq 50$ years). A relatively high proportion of patients ( $\geq 50$ years) receive conservative surgery.

\section{Additional file}

Additional file 1: Table S1. Codes used to define surgery styles. Table S2. Features of patients with borderline ovarian tumors from our hospital during 1996-2017. Figure S1. Flowchart of population selection. Figure

S2. Kaplan-Meier survival curves for all patients with stage I borderline ovarian tumors. (DOCX $134 \mathrm{~kb}$ )

\section{Abbreviations}

AJCC: American joint committee on cancer; BOTs: Borderline ovarian tumors; FIGO: International Federation of Gynecology and Obstetrics; SEER: The surveillance, epidemiology, and end results

Acknowledgements

We thank Dr. Kristin Best for her help in manuscript reviewing.

\section{Funding}

This research is supported by Zhejiang Natural Science Foundation (LY14H160010).

\section{Availability of data and materials}

The datasets generated and analyzed during the current study are available in SEER Program (www.seer.cancer.gov).

\section{Authors' contributions}

SH, YA and WS conceived the concept. SH, YA and WS analyzed data. SH, CX, LN and ZT participated in data collection and interpretation of results. $\mathrm{SH}, \mathrm{ZT}, \mathrm{YA}$ and WS wrote the manuscript. All authors read and approved the final manuscript.

\section{Ethics approval and consent to participate}

This study was exempt from review by the Medical Ethics Committee of Zhejiang Cancer Hospital due to the retrospective nature of the study and data being from a public database.

Consent for publication

Not applicable.

\section{Competing interests}

The authors declare that they have no competing interests.

\section{Publisher's Note}

Springer Nature remains neutral with regard to jurisdictional claims in published maps and institutional affiliations.

\section{Author details}

${ }^{1}$ Department of Gynecologic Oncology, Zhejiang Cancer Hospital, 1 Banshan East Road, Zhejiang 310022, Hangzhou, China. ${ }^{2}$ Department of Gynecology, The First People's Hospital of Aksu, Aksu, China. ${ }^{3}$ Department of Cancer Biology, Wake Forest School of Medicine, Winston Salem, NC 27157, USA.

Received: 17 April 2018 Accepted: 7 June 2018

Published online: 29 June 2018

\section{References}

1. Serov SF, Scully RE, Sobin LH. Histologic typing of ovarian tumors in international histologic classification of tumors (no. 9). Geneva: World Health Organization; 1973. 
2. Seidman JD, Soslow RA, Vang R, Berman JJ, Stoler MH, Sherman ME, et al. Borderline ovarian tumors: diverse contemporary viewpoints on terminology and diagnostic criteria with illustrative images. Hum Pathol. 2004:35:918-33.

3. Benedet JL, Bender H, Jones H 3rd, Ngan HY. Pecorelli S. FIGO staging classifications and clinical practice guidelines in the management of gynecologic cancers. FIGO committee on gynecologic oncology. Int J Gynecol Obstet. 2000;70:209-62.

4. Morice P. Borderline tumours of the ovary and fertility. Eur J Cancer. 2006; 42:149-58.

5. Tinelli R, Tinelli A, Tinelli FG, Cicinelli E, Malvasi A. Conservative surgery for borderline ovarian tumors: a review. Gynecol Oncol. 2006;100:185-91.

6. Skirnisdottir I, Garmo H, Wilander E, Holmberg L. Borderline ovarian tumors in Sweden 1960-2005: trends in incidence and age at diagnosis compared to ovarian cancer. Int J Cancer. 2008;123:1897-901.

7. Akeson M, Zetterqvist BM, Dahllof K, Jakobsen AM, Brannstrom M, Horvath G. Population-based cohort follow-up study of all patients operated for borderline ovarian tumor in western Sweden during an 11-year period. Int J Gynecol Cancer Soc. 2008:18:453-9.

8. Vasconcelos I, de Sousa Mendes M. Conservative surgery in ovarian borderline tumours: a meta-analysis with emphasis on recurrence risk. Eur J Cancer. 2015;51:620-31.

9. Karlsen NMS, Karlsen MA, Hogdall E, Nedergaard L, Christensen IJ, Hogdall C Relapse and disease specific survival in 1143 Danish women diagnosed with borderline ovarian tumours (BOT). Gynecol Oncol. 2016;142:50-3.

10. Alvarez RM, Vazquez-Vicente D. Fertility sparing treatment in borderline ovarian tumours. Ecancermedicalscience. 2015;9:507.

11. Zanetta G, Rota S, Chiari S, Bonazzi C, Bratina G, Mangioni C. Behavior of borderline tumors with particular interest to persistence, recurrence, and progression to invasive carcinoma: a prospective study. J Clin Oncol Off J Am Soc Clin Oncol. 2001;19:2658-64.

12. Morris RT, Gershenson DM, Silva EG, Follen M, Morris M, Wharton JT. Outcome and reproductive function after conservative surgery for borderline ovarian tumors. Obstet Gynecol. 2000;95:541-7.

13. Suh-Burgmann E. Long-term outcomes following conservative surgery for borderline tumor of the ovary: a large population-based study. Gynecol Oncol. 2006;103:841-7.

14. Chen RF, Li J, Zhu TT, Yu HL, Lu X. Fertility-sparing surgery for young patients with borderline ovarian tumors (BOTs): single institution experience. J Ovarian Res. 2016;9:16.

15. Chen X, Fang C, Zhu T, Zhang P, Yu A, Wang S. Identification of factors that impact recurrence in patients with borderline ovarian tumors. J Ovarian Res. 2017;10:23.

16. Uzan C, Kane A, Rey A, Gouy S, Duvillard P, Morice P. Outcomes after conservative treatment of advanced-stage serous borderline tumors of the ovary. Annals of oncology: official journal of the European society for. Med Oncol. 2010;21:55-60.

17. Fischerova D, Zikan M, Dundr P, Cibula D. Diagnosis, treatment, and followup of borderline ovarian tumors. Oncologist. 2012;17:1515-33.

18. Vancraeynest E, Moerman P, Leunen $K$, Amant F, Neven P, Vergote I, Fertility Preservation I. Safe for serous borderline ovarian tumors. Int J Gynecol Cancer. 2016;26:1399-406.

19. Helpman L, Yaniv A, Beiner ME, Aviel-Ronen S, Perri T, Ben-Baruch G, et al. Fertility preservation in women with borderline ovarian tumors - how does it impact disease outcome? A cohort study. Acta Obstet Gynecol Scand. 2017:96:1300-6.

20. Trillsch F, Mahner S, Woelber L, Vettorazzi E, Reuss A, Ewald-Riegler N, et al. Agedependent differences in borderline ovarian tumours (BOT) regarding clinical characteristics and outcome: results from a sub-analysis of the Arbeitsgemeinschaft Gynaekologische Onkologie (AGO) ROBOT study. Annals of oncology: official journal of the European society for. Med Oncol. 2014;25:1320-7.

21. Surveillance $E$, and End Results (SEER) Program. (www.seer.cancer.gov) SEER*Stat Database: Incidence - SEER 18 Regs Research Data + Hurricane Katrina Impacted Louisiana Cases, Nov 2016 Sub (1973-2014 varying) Linked To County Attributes - Total U.S., 1969-2015 Counties, National Cancer Institute, DCCPS, Surveillance Research Program, Surveillance Systems Branch, released April 2017, based on the November 2016 submission.

22. Sherman ME, Mink PJ, Curtis R, Cote TR, Brooks S, Hartge P, et al. Survival among women with borderline ovarian tumors and ovarian carcinoma: a population-based analysis. Cancer. 2004;100:1045-52.
23. Matsuo K, Machida H, Takiuchi T, Grubbs BH, Roman LD, Sood AK, et al. Role of hysterectomy and lymphadenectomy in the management of early-stage borderline ovarian tumors. Gynecol Oncol. 2017;144:496-502.

24. Beahrs OH, Henson DE, Hutter RVP, Myers MH, editors. AJCC manual for staging of cancer. 3rd ed. Philadelphia: JB Lippincott; 1988. P. 163-8.

25. Prat J, FIGO committee on gynecologic oncology. Staging classification for cancer of the ovary, fallopian tube, and peritoneum. Int J Gynecol Obstet. 2014;124:1-5.

26. Trillsch F, Mahner S, Ruetzel J, Harter P, Ewald-Riegler N, Jaenicke F, et al. Clinical management of borderline ovarian tumors. Expert Rev Anticancer Ther. 2010;10:1115-24.

27. Longacre TA, McKenney JK, Tazelaar HD, Kempson RL, Hendrickson MR. Ovarian serous tumors of low malignant potential (borderline tumors): outcome-based study of 276 patients with long-term ( $>$ or $=5$-year) followup. Am J Surg Pathol 2005; 29:707-723.

28. Wong HF, Low JJ, Chua Y, Busmanis I, Tay EH, Ho TH. Ovarian tumors of borderline malignancy: a review of 247 patients from 1991 to 2004. Int J Gynecol Cancer. 2007;17:342-9.

29. du Bois A, Ewald-Riegler N, de Gregorio N, Reuss A, Mahner S, Fotopoulou $C$, et al. Borderline tumours of the ovary: a cohort study of the Arbeitsgmeinschaft Gynakologische Onkologie (AGO) study group. Eur J Cancer. 2013:49:1905-14.

30. Seong SJ, Kim DH, Kim MK, Song T. Controversies in borderline ovarian tumors. J Gynecol Oncol. 2015;26:343-9.

31. Kaern J, Trope CG, Kristensen GB, Abeler VM. Pettersen EO. DNA ploidy; the most important prognostic factor in patients with borderline tumors of the ovary. Int J Gynecol Cancer. 1993;3:349-58.

32. Malpica A, Wong KK. The molecular pathology of ovarian serous borderline tumors. Ann Oncol. 2016;27(Suppl 1):i16-i9.

33. Anglesio MS, Kommoss S, Tolcher MC, Clarke B, Galletta L, Porter H, et al. Molecular characterization of mucinous ovarian tumours supports a stratified treatment approach with HER2 targeting in 19\% of carcinomas. J Pathol. 2013:229:111-20.

34. Mackenzie R, Kommoss S, Winterhoff BJ, Kipp BR, Garcia JJ, Voss J, et al. Targeted deep sequencing of mucinous ovarian tumors reveals multiple overlapping RAS-pathway activating mutations in borderline and cancerous neoplasms. BMC Cancer. 2015:15:415.

35. Levi F, La Vecchia C, Randimbison L, Te VC. Borderline ovarian tumours in Vaud, Switzerland: incidence, survival and second neoplasms. Br I Cancer. 1999;79:4-6.

36. Lesieur B, Kane A, Duvillard P, Gouy S, Pautier P, Lhomme C, et al. Prognostic value of lymph node involvement in ovarian serous borderline tumors. Am J Obstet Gynecol. 2011;204:438 e1-7.

37. McKenney JK, Balzer BL, Longacre TA. Lymph node involvement in ovarian serous tumors of low malignant potential (borderline tumors): pathology, prognosis, and proposed classification. Am J Surg Pathol. 2006:30:614-24.

38. Djordjevic B, Malpica A. Ovarian serous tumors of low malignant potential with nodal low-grade serous carcinoma. Am J Surg Pathol. 2012;36:955-63.

39. Lazarou A, Fotopoulou C, Coumbos A, Sehouli J, Vasiljeva J, Braicu I, et al. Long-term follow-up of borderline ovarian tumors clinical outcome and prognostic factors. Anticancer Res. 2014;34:6725-30.

40. Cusido M, Balaguero L, Hernandez G, Falcon O, Rodriguez-Escudero FJ, Vargas JA, et al. Results of the national survey of borderline ovarian tumors in Spain. Gynecol Oncol. 2007;104:617-22.

41. Leake JF, Currie JL, Rosenshein NB, Woodruff JD. Long-term follow-up of serous ovarian tumors of low malignant potential. Gynecol Oncol. 1992;47:150-8.

\section{Ready to submit your research? Choose BMC and benefit from:}

- fast, convenient online submission

- thorough peer review by experienced researchers in your field

- rapid publication on acceptance

- support for research data, including large and complex data types

- gold Open Access which fosters wider collaboration and increased citations

- maximum visibility for your research: over $100 \mathrm{M}$ website views per year

At BMC, research is always in progress.

Learn more biomedcentral.com/submissions 